University of Wollongong

Research Online

Australian Institute for Innovative Materials -

Papers

Australian Institute for Innovative Materials

$1-1-2013$

Limiting thermomagnetic avalanches in superconducting films by stopholes

F Colauto

Federal University Of Sao Carlos, Brazil

J I. Vestgarden

University of Oslo

A M H De Andrade

Universidade Federal Do Rio Grande Do Sul

A A M Oliveira

Federal University Of Sao Carlos, Brazil

Wilson Aires Ortiz

Federal University Of Sao Carlos, Brazil

See next page for additional authors

Follow this and additional works at: https://ro.uow.edu.au/aiimpapers

Part of the Engineering Commons, and the Physical Sciences and Mathematics Commons

Research Online is the open access institutional repository for the University of Wollongong. For further information contact the UOW Library: research-pubs@uow.edu.au 


\title{
Limiting thermomagnetic avalanches in superconducting films by stop-holes
}

\author{
Abstract \\ It is demonstrated that circular holes in superconducting films of $\mathrm{Nb}$ can arrest the propagation of \\ thermomagnetic avalanches. The effect was found over a range of temperatures where the material is \\ susceptible to this instability. For other hole shapes, like square and triangular, the sharp corners provoke \\ secondary avalanches, thus extending the breakdown. Making use of circular stop-holes can become a \\ practical way to limit thermomagnetic breakdown in superconducting films. \\ Keywords \\ stop, films, holes, superconducting, limiting, avalanches, thermomagnetic \\ Disciplines \\ Engineering | Physical Sciences and Mathematics \\ Publication Details \\ Colauto, F., Vestgarden, J. I., De Andrade, A. M H., Oliveira, A. A M., Ortiz, W. Aires. and Johansen, T. H. \\ (2013). Limiting thermomagnetic avalanches in superconducting films by stop-holes. Applied Physics \\ Letters, 103 (3), 032604-1-032604-4.

\section{Authors} \\ F Colauto, J I. Vestgarden, A M H De Andrade, A A M Oliveira, Wilson Aires Ortiz, and Tom H. Johansen
}




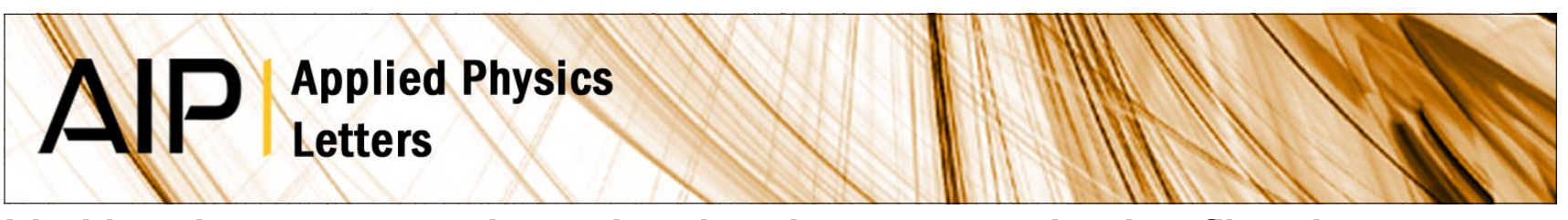

\section{Limiting thermomagnetic avalanches in superconducting films by stop- holes}

F. Colauto, J. I. Vestgården, A. M. H. de Andrade, A. A. M. Oliveira, W. A. Ortiz et al.

Citation: Appl. Phys. Lett. 103, 032604 (2013); doi: 10.1063/1.4813908

View online: http://dx.doi.org/10.1063/1.4813908

View Table of Contents: http://apl.aip.org/resource/1/APPLAB/v103/i3

Published by the AIP Publishing LLC.

\section{Additional information on Appl. Phys. Lett.}

Journal Homepage: http://apl.aip.org/

Journal Information: http://apl.aip.org/about/about_the_journal

Top downloads: http://apl.aip.org/features/most_downloaded

Information for Authors: http://apl.aip.org/authors

\section{ADVERTISEMENT}

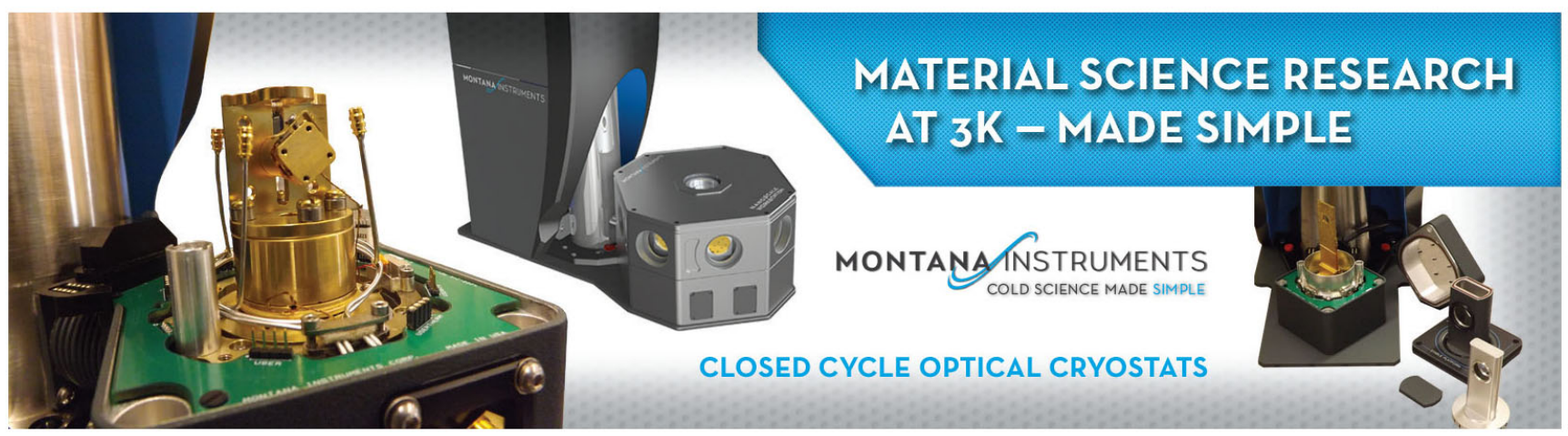




\title{
Limiting thermomagnetic avalanches in superconducting films by stop-holes
}

\author{
F. Colauto, ${ }^{1}$ J. I. Vestgården, ${ }^{2}$ A. M. H. de Andrade, ${ }^{3}$ A. A. M. Oliveira, ${ }^{1,4}$ W. A. Ortiz, ${ }^{1}$ \\ and T. H. Johansen 2,5 \\ ${ }_{1}^{1}$ Departamento de Física, Universidade Federal de São Carlos, 13565-905 São Carlos, SP, Brazil \\ ${ }^{2}$ Department of Physics, University of Oslo, P.O. Box 1048 Blindern, 0316 Oslo, Norway \\ ${ }^{3}$ Instituto de Física, Universidade Federal do Rio Grande do Sul, 91501-970 Porto Alegre, RS, Brazil \\ ${ }^{4}$ Instituto Federal de Educação, Ciência e Tecnologia de São Paulo-Campus Matão, 15990-040 Matão, SP, \\ Brazil \\ ${ }^{5}$ Institute for Superconducting and Electronic Materials, University of Wollongong, Northfields Avenue, \\ Wollongong, NSW 2522, Australia
}

(Received 5 June 2013; accepted 28 June 2013; published online 16 July 2013)

\begin{abstract}
It is demonstrated that circular holes in superconducting films of $\mathrm{Nb}$ can arrest the propagation of thermomagnetic avalanches. The effect was found over a range of temperatures where the material is susceptible to this instability. For other hole shapes, like square and triangular, the sharp corners provoke secondary avalanches, thus extending the breakdown. Making use of circular stop-holes can become a practical way to limit thermomagnetic breakdown in superconducting films. (C) 2013 AIP Publishing LLC. [http://dx.doi.org/10.1063/1.4813908]
\end{abstract}

Successful performance of devices based on type-II superconductor components relies fully upon the thermomagnetic stability of the superconducting material. When vortices, lines carrying one quantum of magnetic flux are formed, the stability depends on how strongly they are pinned by inhomogeneities created naturally during the material synthesis or made intentionally at a later stage. In such systems a runaway can develop, e.g., when a temperature fluctuation makes the pinning decrease locally, immediately allowing some flux to move. Dissipation from the vortex displacements causes the temperature to rise even further, and unless compensated by internal heat diffusion or heat removal to surroundings, an avalanche of magnetic flux motion and dissipation will take place. During such events the superconductor can be heated to the flux-flow state, or even above its critical temperature, see Ref. 1 for a review.

In films, this instability leads to formation of finger-like flux structures, as found both theoretically, ${ }^{2}$ and experimentally in many materials of technological importance. ${ }^{3}$ Magnetooptical imaging (MOI) experiments have shown that the flux dendrites can grow during the runaway to lengths on the order of the sample size in approximately $100 \mathrm{~ns} .{ }^{4}$ When triggered by an increasing external magnetic field, the avalanches nucleate and start propagating from the film edge where the exact starting point is essentially unpredictable, since disorder like edge roughness plays a key role. Due to disorder also within the film, the detailed propagation route of an avalanche is unpredictable too. Moreover, quite often one finds that during a runaway the avalanche splits into two or more branches. Indeed, the dendritic patterns formed in this process show striking resemblance to those seen in atmospheric lightning, ${ }^{5}$ and also to those formed during the propagation of mechanical cracks. As for the transient behavior, the thermomagnetic runaways resemble lightning by the rapid decay of the increased temperature and electrical field, while they resemble cracking by leaving permanent fingerprints of the damage by trapping the flux entering the sample during an avalanche. Importantly, the latter implies also permanent rerouting of the flow of supercurrents. ${ }^{5}$
Making holes of proper size in superconducting films is a well-known approach for arresting the motion of individual vortices. ${ }^{6}$ Indeed, it has been demonstrated in superconductorbased devices, e.g., rf-SQUIDs and microwave resonators, that noise can be reduced significantly by adding holes at strategic locations in the film structure. ${ }^{7}$

In this work, we investigate whether holes can have a stabilizing effect on the far more dramatic thermomagnetic avalanches by limiting, or possibly stopping the propagation of these self-propelled runaways. A similar idea is known to work very well for mechanical fracture fronts propagating in plates, where drilling a hole can arrest a crack front from further advancement. ${ }^{8}$ By making a hole in a superconducting film, one creates a surface to distribute the load, i.e., the magnetic pressure, should an avalanche strike there. Thus, stabilizing effects using properly shaped stop-holes can be expected. We explore here this idea by using MOI to observe the flux dynamics in films of $\mathrm{Nb}$ patterned with holes of different shapes.

Samples of $\mathrm{Nb}$ were prepared as films of thickness $200 \mathrm{~nm}$ by magnetron sputtering in a UHV system with base pressure lower than $2 \times 10^{-8}$ Torr. The films were shaped using optical lithography into squares with sides $2.5 \mathrm{~mm}$, and patterned with one hole at a distance of $0.25 \mathrm{~mm}$ from each edge. The typical hole size is $0.5 \mathrm{~mm}$, i.e., larger than the typical width of an individual branch in an avalanche, thus allowing a hole to easily capture an approaching flux finger. The holes were shaped as circles, squares, and triangles to explore how the stop-hole effect depends on geometrical aspects.

The MOI was performed using a Faraday rotating Bisubstituted ferrite-garnet film with in-plane magnetization as sensor. ${ }^{9}$ Placing the sensor directly on the superconducting film, the flux density distribution was detected as an image in a polarized light microscope. ${ }^{10}$ We used slightly uncrossed polarizers allowing to distinguish between flux parallel and antiparallel to the applied field. In the shown images zero flux density, e.g., as in the Meissner state, is represented by an intermediate brightness level. Excess brightness represents the 
density of flux parallel to the applied field, whereas darker contrast indicates reverse flux. The zig-zag lines seen in most of the presented images are Bloch walls in the garnet film. Whereas their motion can be used to manipulate individual vortices, ${ }^{11}$ their effect on the present large-scale experiments is negligible. A resistive coil was used to apply a uniform magnetic field perpendicular to the sample.

The high quality of the sample is evidenced by the image in Fig. 1, showing the remanent state of a film patterned with 4 circular holes. Initially, the applied field was raised to $\mu_{0} H_{a}=4.8 \mathrm{mT}$, which at $8 \mathrm{~K}$ is sufficiently large to create a subsequent remanent state where the critical current circulates the whole sample in one direction. The resulting flux distribution seen here reveals an excellent material uniformity and successful patterning process. The set of bright lines shows the expected combination of diagonal and parabolic lines (d-lines) where sharp turns take place in the flow of critical current. ${ }^{10}$

Shown in Fig. 2 (upper) is a magneto-optical image of the flux distribution in the same film, first zero-field-cooled to $2.5 \mathrm{~K}$, and then exposed to an applied field of $1.85 \mathrm{mT}$. Here one sees that flux barely penetrates the sample, causing piling up of field around the external edge. Evidently, also the holes give contrast and thus contain flux, in spite they are fully embedded in the Meissner-state area. Positive (bright) and negative (dark) flux concentrate near each hole's inner and outer side, respectively. This dipolar feature arises from the perturbation of the Meissner-state currents as they split to flow around each hole, ${ }^{12}$ which imposes opposite curvatures in the otherwise parallel stream lines, as indicated in the figure.

After slowly ramping the applied field to $2.10 \mathrm{mT}$, the flux distribution becomes as seen in Fig. 2 (lower). Three avalanches have now occurred, all starting near the midpoint of a side from where they have channeled flux into the nearest hole. Flux is now piling up at the inner edge of the holes. Most importantly, the image shows that the holes prevent further propagation of the runaway.

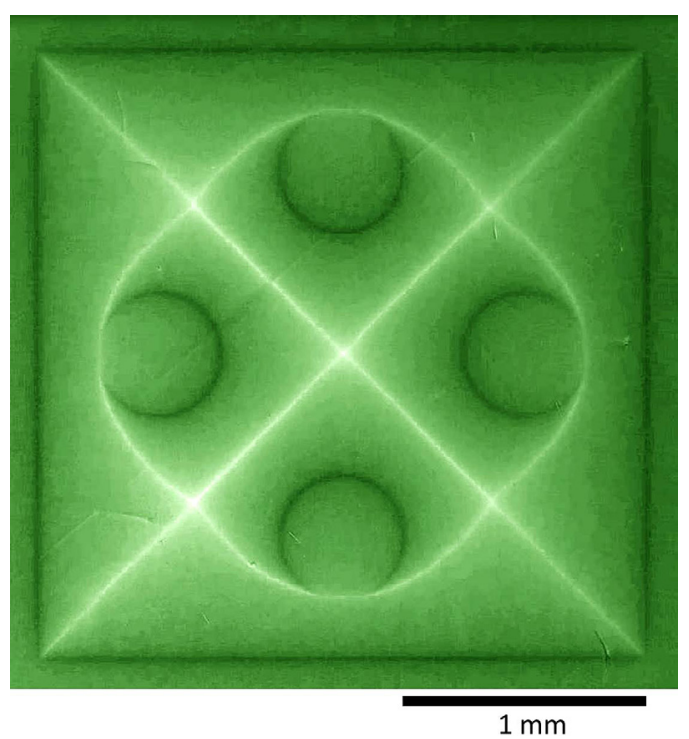

FIG. 1. Magneto-optical image of the perpendicular flux density in a $\mathrm{Nb}$ film with 4 circular holes brought to the remanent state after first applying a large magnetic field at $8 \mathrm{~K}$. The flawless symmetric image shows that the patterned film has an excellent overall uniformity.
To compare the effect of a circular hole with that of holes of other shapes, similar samples were patterned with one circular hole in combination with square and triangular ones. Shown in Fig. 3 (upper) is an image of the flux distribution after first zero-field-cooling the sample to $2.4 \mathrm{~K}$, and then applying $2.25 \mathrm{mT}$. At this field, all the holes have been filled with flux via respective avalanches starting from the sample rim. Evidently, the square and triangular holes generate secondary avalanches from their inner corners, whereas the circular hole also here resists such continued propagation. This can be understood from the fact that the magnitude of the electrical field is a key parameter deciding the onset of the thermomagnetic instability. ${ }^{2}$ Since corners being concave with respect to the current flow pattern are indeed locations of largely enhanced electrical fields, ${ }^{13}$ the superior stability of the circular hole is in full accordance with expectations.

Increasing the field further to $3.15 \mathrm{mT}$ (middle panel) generates nine more avalanches, all starting from the sample rim and ending blindly inside the Meissner-state area without perturbing any of the holes. Noticeable on the image is the enhanced brightness of all these new avalanches as compared to those that channeled flux into the holes. The lower panel of Fig. 3 shows the flux distribution from another run
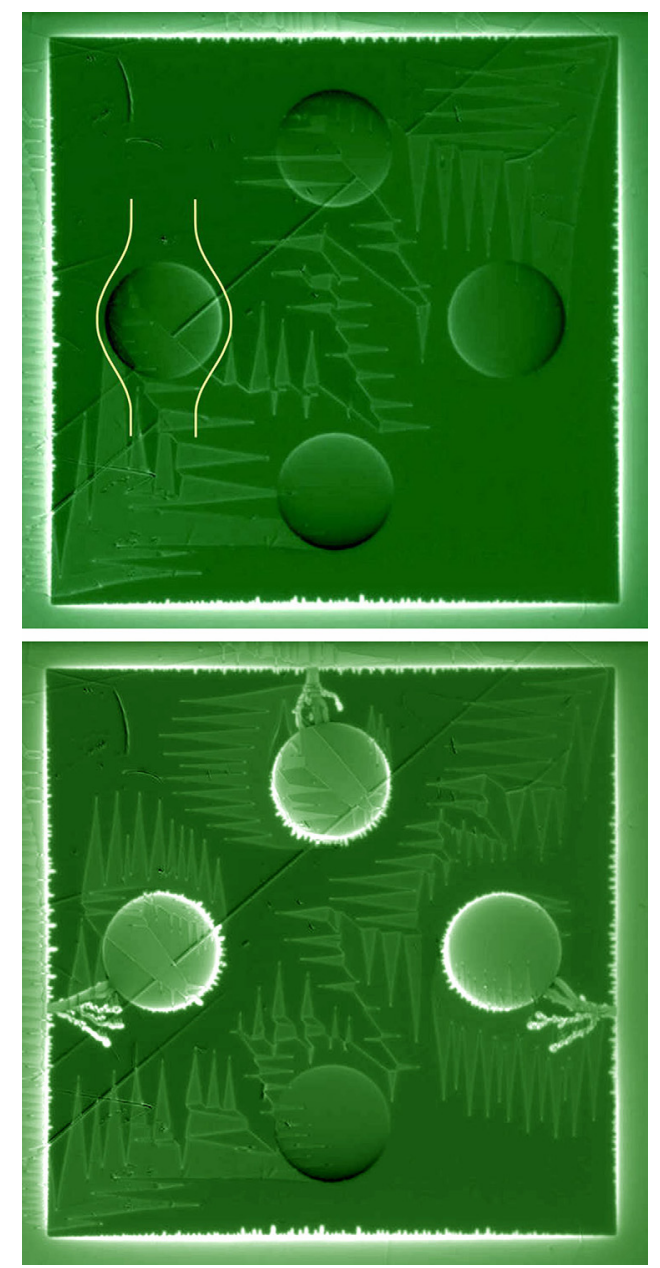

FIG. 2. Top: Image taken after initial zero-field cooling to $2.5 \mathrm{~K}$, and then increasing the field to $1.85 \mathrm{mT}$. Bottom: Increasing the field further to $2.10 \mathrm{mT}$ triggers 3 avalanches pumping flux into 3 of the holes. All 4 holes are nominally identical, but do not become filled with flux simultaneously due to microscopic disorder. 

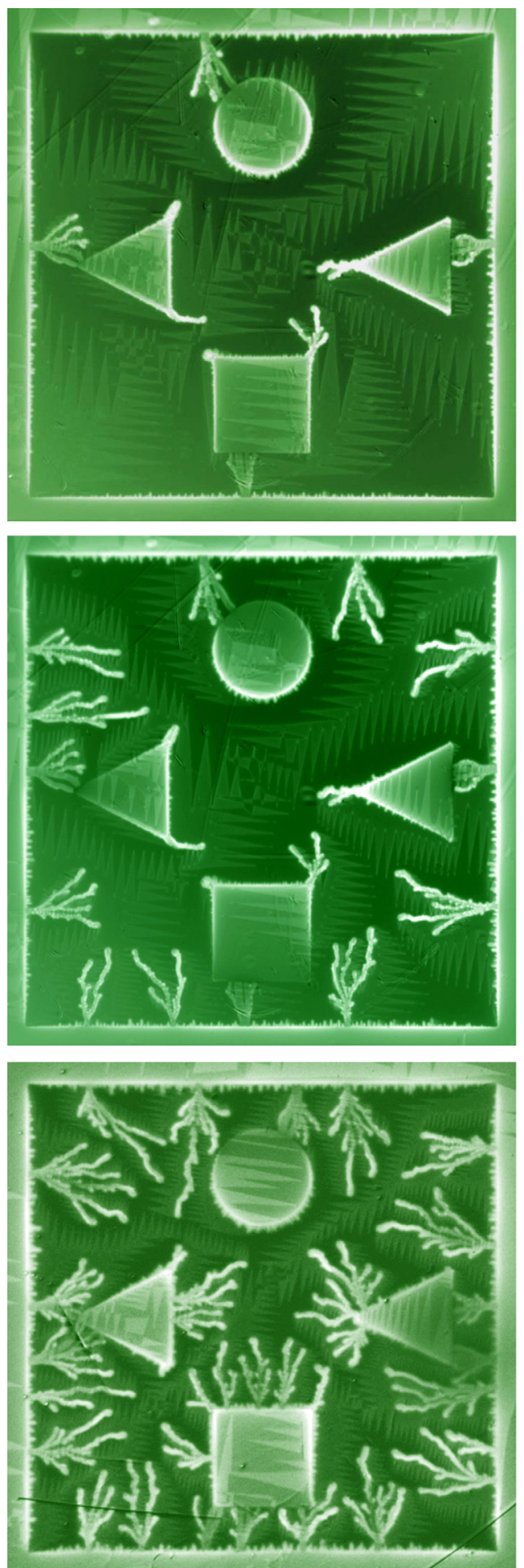

FIG. 3. Images of the flux distribution in a sample with differently shaped holes. After initial zero-field cooling, the images were taken at applied fields of $2.25 \mathrm{mT}$ (upper), $3.15 \mathrm{mT}$ (middle), and $4.20 \mathrm{mT}$ (lower).

at $2.5 \mathrm{~K}$, where a field of $4.20 \mathrm{mT}$ was applied. In spite of the massive invasion of avalanches at this field, the circular hole again demonstrates a clear stop-hole effect.

To explain the distinct difference in the brightness of avalanche branches feeding flux into a hole, and those ending blindly elsewhere in the film, see Fig. 4, we propose the following scenario. Before an avalanche hits a hole, substantial amounts of reverse flux are concentrated in the part of the hole facing the nearest external sample edge, as evident from
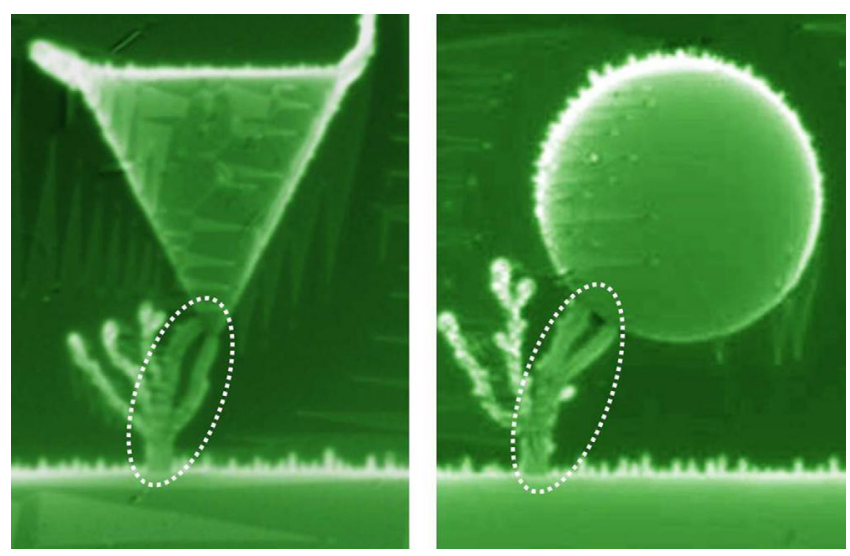

FIG. 4. Images showing dark dendritic channels that have pumped flux into holes and bright branches ending blindly outside. These examples are magnified views from Figs. 2 (lower) and 3 (upper).

Fig. 2. Then, when an avalanche suddenly punctures the hole, the invading flux will immediately meet the antiflux inside the hole, and annihilation will take place. Thus, when the event is over, and the magnetic pressures from both outside and inside the hole are equilibrated, the overall flux density in the flux-feeding branches has reduced. This recombination process does not take place in the branches ending blindly, and thus they appear brighter in the images.

Shown in Fig. 5 are the results of an investigation at a higher temperature of $4.5 \mathrm{~K}$, of a film patterned into four samples where three contain different hole patterns while one is plain. After initial zero-field cooling, a field of $2.6 \mathrm{mT}$ was here applied. At this field, all the samples have been invaded by flux avalanches. In the upper left one, containing four square holes, an avalanche started from the right edge filling the nearest hole, and then moved on via two branched structures from inner corners, hitting the nearest holes which also became filled with flux. In the upper right sample patterned with only triangular holes, an event started at the lower edge, and created secondary avalanches at both inner corners. In the lower right sample, two of the holes were filled with flux via avalanches. Here, the circular hole again demonstrated the stop-hole effect, in stark contrast to the square hole which

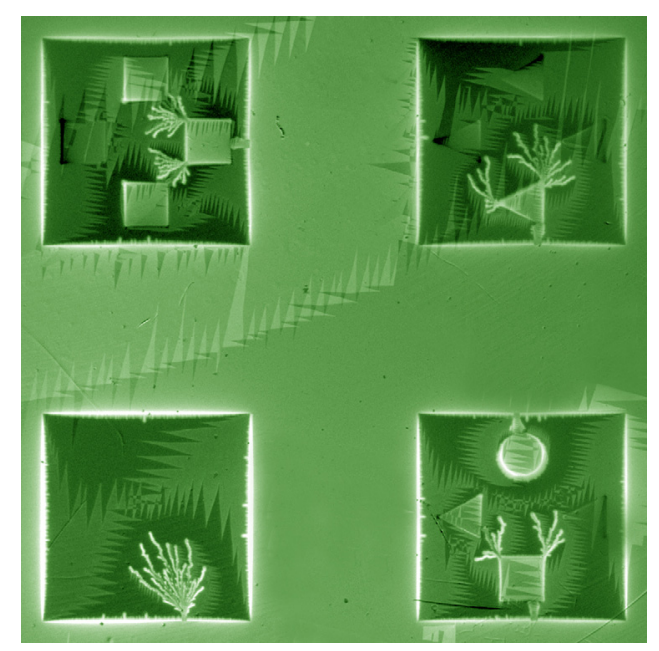

FIG. 5. Image of the flux distribution at $4.5 \mathrm{~K}$ in a $\mathrm{Nb}$ film consisting four samples of side $2.5 \mathrm{~mm}$. It reveals a stabilizing effect of the circular hole. Holes with corners show a strong tendency to generate secondary avalanches. 
continued generating secondary events. The avalanche seen in the lower left sample displays a typical event in an unpatterned film, and illustrates that the region which is perturbed in such an avalanche can be more extensive than when a circular hole captures the invading flux.

In summary, we have shown that circular holes in superconducting films can arrest the propagation of thermomagnetic avalanches. The effect was seen over a range of temperatures where the material is susceptible to this instability. For other hole shapes, like square and triangular, the sharp corners easily provoke secondary instabilities, thus extending the size of the avalanche. While the shown images could suggest that circular holes never generate secondary avalanches, we emphasize that they indeed do. However, this happens only at fields larger than those triggering avalanches when square and triangular holes are present. After a careful optimization of geometrical aspects, the use of stopholes can become a practical way to limit thermomagnetic breakdown in superconducting films.

The samples were grown in Laboratório de Conformação Nanométrica (LCN-IF-UFRGS), and the lithography was made in Laboratório de Microfabricação (LNNano/CNPEM). The work was partially supported by the Brazilian funding agencies FAPESP and CNPq, the Brazilian program Science without Borders, and by the Norwegian Research Council.

${ }^{1}$ R. G. Mints and A. L. Rakhmanov, Rev. Mod. Phys. 53, 551 (1981).

${ }^{2}$ A. L. Rakhmanov, D. V. Shantsev, Y. M. Galperin, and T. H. Johansen, Phys. Rev. B 70, 224502 (2004); D. V. Denisov, A. L. Rakhmanov,
D. V. Shantsev, Y. M. Galperin, and T. H. Johansen, Phys. Rev. B 73, 014512 (2006).

${ }^{3}$ P. Leiderer, J. Boneberg, P. Brüll, V. Bujok, and S. Herminghaus, Phys. Rev. Lett. 71, 2646 (1993); C. A. Durán, P. L. Gammel, R. E. Miller, and D. J. Bishop, Phys. Rev. B 52, 75 (1995); T. H. Johansen et al., Europhys. Lett. 59, 599 (2002); I. A. Rudnev, S. V. Antonenko, D. V. Shantsev, T. H. Johansen, and A. E. Primenko, Cryogenics 43, 663 (2003); S. C. Wimbush, B. Holzapfel, and Ch. Jooss, J. Appl. Phys. 96, 3589 (2004); I. A. Rudnev, D. V. Shantsev, T. H. Johansen, and A. E. Primenko, Appl. Phys. Lett. 87, 042502 (2005); E.-M. Choi et al., Appl. Phys. Lett. 87, 152501 (2005); M. Motta et al., Appl. Phys. Lett. 102, 212601 (2013).

${ }^{4}$ U. Bolz, B. Biehler, D. Schmidt, B. Runge, and P. Leiderer, Europhys. Lett. 64, 517 (2003).

${ }^{5}$ J. I. Vestgården, D. V. Shantsev, Y. M. Galperin, and T. H. Johansen, Sci. Rep. 2, 886 (2012).

${ }^{6}$ V. V. Moshchalkov, M. Baert, V. V. Metlushko, E. Rosseel, M. J. Van Bael, K. Temst, Y. Bruynseraede, and R. Jonckheere, Phys. Rev. B 57, 3615 (1998).

${ }^{7}$ P. Selders and R. Woerdenweber, IEEE Trans. Appl. Supercond. 11, 928 (2001); D. Bothner, C. Clauss, E. Koroknay, M. Kemmler, T. Gaber, M. Jetter, M. Scheffler, P. Michler, M. Dressel, D. Koelle, and R. Kleiner, Appl. Phys. Lett. 100, 012601 (2012).

${ }^{8}$ H. Miyagawa and H. Nisitani, Bull. JSME 28, 2219 (1985).

${ }^{9}$ L. E. Helseth, R. W. Hansen, E. I. Il'yashenko, M. Baziljevich, and T. H. Johansen, Phys. Rev. B 64, 174406 (2001); T. H. Johansen, M. Baziljevich, H. Bratsberg, Y. Galperin, P. E. Lindelof, Y. Shen, and P. Vase, Phys. Rev. B 54, 16264 (1996).

${ }^{10}$ Ch. Jooss, J. Albrecht, H. Kuhn, S. Leonhardt, and H. Kronmüller, Rep. Prog. Phys. 65, 651 (2002).

${ }^{11}$ P. E. Goa, H. Hauglin, A. A. F. Olsen, D. Shantsev, and T. H. Johansen, Appl. Phys. Lett. 82, 79 (2003).

${ }^{12}$ M. Baziljevich, T. H. Johansen, H. Bratsberg, Y. Galperin, P. E. Lindelhof, Y. Shen, and P. Vase, Appl. Phys. Lett. 69, 3590 (1996).

${ }^{13}$ T. Schuster, H. Kuhn, and E. H. Brandt, Phys. Rev. B 54, 3514 (1996); J. I. Vestgården and T. H. Johansen, Supercond. Sci. Technol. 25, 104001 (2012). 